\title{
Diagnosis and Management of Latent Tuberculosis Infection
}

\author{
Laura Muñoz ${ }^{1,2}$, Helen R. Stagg ${ }^{2}$, and Ibrahim Abubakar ${ }^{2}$ \\ ${ }^{1}$ Infectious Diseases Department, Bellvitge University Hospital-IDIBELL, Barcelona 08970, Spain \\ ${ }^{2}$ Research Department of Infection and Population Health, University College London, London \\ WC1E 6JB, United Kingdom \\ Correspondence: i.abubakar@ucl.ac.uk
}

\begin{abstract}
The post-2015 World Health Organization global tuberculosis strategy recognizes that elimination requires a focus on reducing the pool of latently infected individuals, an estimated $30 \%$ of the global population, from which future tuberculosis cases would be generated. Tackling latent tuberculosis infection requires the identification and treatment of asymptomatic individuals to reduce the risk of progression to active disease. Diagnosis of latent tuberculosis infection is based on the detection of an immune response to Mycobacterium tuberculosis antigens using either the tuberculin skin test or interferon- $\gamma$ release assays. Current treatment requires the use of antibiotics for at least 3 months. In this article, we review the current knowledge of the natural history, immunology, and pathogenesis of latent tuberculosis, describe key population groups for screening and risk assessment, discuss clinical management in terms of diagnosis and preventative treatment, and identify areas for future research.
\end{abstract}

atent tuberculosis infection (LTBI) is defined by the detection of a specific immune response to Mycobacterium tuberculosis complex (MTC) antigens in a healthy subject (i.e., with no symptoms or signs of active tuberculosis [TB]). As M. tuberculosis can only be isolated from humans when it is in an active phase, causing illness, the detection of the presence of LTBI is wholly reliant on indirect measurements of immune reactivity to antigenic challenge.

By evading both innate and adaptive immunity, bacteria of the MTC are able to persist in a dormant phase for several decades, or even for the lifetime of the host. In $\sim 10 \%$ of all infected individuals, a LTBI will progress to active replication and cause TB disease. This can be prevented with antibiotic treatment; globally standard regimens are of 6-9-mo duration with a single drug, or at least 3 mo of two antibiotics. Increasing the number of drugs within the regimen increases the potential for consequent adverse events. Given the small proportion of LTBI-positive individuals who develop active $\mathrm{TB}$, the imperfect effectiveness of LTBI treatment and associated its side effects, the critical question is, therefore, who should be targeted for treatment? This question is pertinent because none of the tests currently available are

Editors: Stefan H.E. Kaufmann, Eric J. Rubin, and Alimuddin Zumla

Additional Perspectives on Tuberculosis available at www.perspectivesinmedicine.org

Copyright (C) 2015 Cold Spring Harbor Laboratory Press; all rights reserved; doi: 10.1101/cshperspect.a017830

Cite this article as Cold Spring Harb Perspect Med 2015;5:a017830 
able to accurately predict future progression. It is widely recognized that the risk of progression is highest in young children, the immunosuppressed, and shortly after infection. Pragmatically, therefore, these individuals should be targeted for LTBI screening and treatment. In most cases, this would lead to an "intention to test is intention to treat" approach, with an exception for close contacts of multidrug-resistant TB cases, in which testing may be used to inform "watchful waiting."

In this article, we review the current knowledge of the natural history and immunology of LTBI, describe key population groups for screening and risk assessment, discuss clinical management in terms of diagnosis and preventative treatment, and finally look to the key fields for future research.

\section{HISTORY (CONCEPT OF DORMANT BACILLI)}

Although TB is one of the most ancient diseases, LTBI was only recognized at the end of the 19th century following (1) the accurate description of the morbid anatomy of TB, its clinical evolution, and surrounding circumstances; (2) the first experimental animal models; and (3) the development of essential tools such as the microscope. Soon after the discovery of the causative agent of TB in 1882, Robert Koch created the Koch's fluid ("a glycerine extract of pure cultivations of tubercle bacilli" [Koch 1891]), for use as a live vaccine; it turned out to be an ineffective preventive agent. Koch's fluid, which was given the name of tuberculin, later proved to be a valuable diagnostic tool for active TB in cattle. In the last years of the 19th century, experiments showed that cows with confirmed TB developed a febrile reaction $14-18 \mathrm{~h}$ after tuberculin administration. Apparently healthy cows who presented the same systemic reaction were slaughtered; some of the necropsies revealed active tubercles (Mi'fadyean 1891; Martin and Robins 1898). Reactive cattle without tubercles had their positive reaction attributed to dormant TB, as quiescent tuberculous foci were identified in some of the necropsies (Martin and Robins 1898).
Mantoux's description of a new technique to administer tuberculin (the "tuberculin skin test" [TST]) in 1908, together with its improved composition in 1934 (purified protein derivate [PPD]), made its use as a human diagnostic method possible, as systemic reactions were not observed in tested subjects. The first trials that tested tuberculin on TB patients also showed positive results in healthy controls. It was at this time that the notion of latent bacteria or bacillary allergy emerged.

As for preventive purposes, the 20th century saw the introduction of several successful vaccines, and there were great expectations for an effective preparation against TB. In 1902, Behring proposed to use the bacillus isolated from humans for the vaccination of cows. Calmette and Guerin passaged Mycobacterium bovis, which has antigenic properties similar to those of the virulent $M$. tuberculosis bacilli and the ability to provoke antibody formation in humans, to obtain the attenuated Bacillus Calmette-Guérin (BCG) strain. BCG was successfully tested in infants exposed to family cases of TB owing to the high mortality usually seen in such infants (72\%) (Calmette 1931).

The immunological response to M. bovis and $M$. tuberculosis is highly similar, allowing the effective use of BCG as a vaccine and tuberculin as a diagnostic reagent. The proportion of BCG-vaccinated people who have a positive tuberculin skin test (TST) has been reported to vary from $0 \%$ to $90 \%$ (Wang et al. 2002). These wide differences may depend on dose, the manufacturer of the vaccine, age, and the interval between vaccination and testing. Variation in the cutoff for positivity used during TST testing also explains some of the observed variation. Nevertheless, a positive reaction of $>15 \mathrm{~mm}$ in someone who was vaccinated $>15 \mathrm{yr}$ before is unlikely to be related to BCG vaccination and would likely reflect the presence of TB infection.

Questions were raised about the infectiousness of the tubercle bacilli and how to prevent the spread of the disease. This led to early contact studies surrounding single active cases, with the aim of finding latently infected individuals ( positive TST responders but without TB symptoms) and treating them to prevent the devel- 
opment of active TB. Soon after the discovery of streptomycin (1944) and isoniazid (1952) for active TB, the first experiments using isoniazid as a preventive treatment in an animal model were undertaken (1955). Initial clinical trials in humans began in 1957, with results reported in the mid-1960s. During the next few decades, five major randomized trials showed the benefits of isoniazid in preventing active TB.

\section{NATURAL HISTORY AND PATHOGENESIS}

Underpinning all attempts at the diagnosis and treatment of LTBI is our understanding of the natural history of $\mathrm{TB}$, particularly the process through which LTBI is established, and the host and bacterial factors influential in determining latency and progression. The most important reservoir of $M$. tuberculosis is humans, whereas cattle are the equivalent host for M. bovis. Zoonotic transmission of $M$. bovis was frequent in ancient times; however, pasteurization of milk and the testing of herds decreased the public health impact of M. bovis in humans in wealthier countries. Many developing countries continue to observe zoonotic transmission.

Humans with active pulmonary TB currently play the dominant role in the transmission and maintenance of TB. Studies by Flügge in 1899 (Hillier 1903) showed that both dried tuberculous sputum dust and the minute drops coughed out by pulmonary TB patients were capable of surviving for several days while remaining infectious. These tiny particles contain tubercle bacilli inside droplet nuclei, are easily inhaled, and constitute the route of entry and first contact with the healthy subject (Box 1).

\section{Infection}

On inhalation, $M$. tuberculosis reaches the main airway and alveoli and faces the first cell-mediated barrier of the innate immune system. Alveolar macrophages phagocytose the pathogen and isolate it through a process of membrane invaginations that finally culminates in phagosome formation. From now on, two contending forces compete for control-the bacterium's struggle for survival and macrophage's attempts to contain the infection.

After phagosome formation, infected macrophages and dendritic cells display M. tuberculosis antigens via major histocompatibility complex (MHC) class II molecules. These professional antigen-presenting cells expressing MTC molecules travel to the lymph system and then into local mediastinal lymph nodes, where they will activate $\mathrm{CD} 4^{+}$T-helper cells (Th). These cells regulate the secretion of cytokines via endocrine and paracrine signaling and function in the formation and maintenance of granulomas by means of a proinflammatory response signal cascade (interferon- $\gamma$ [IFN- $\gamma$ ], interleukin-2 [IL-2], and tumor necrosis factor- $\alpha[$ TNF- $\alpha]$ ), which leads to the attraction of mononuclear cells and T lymphocytes. These cell types, together with B lymphocytes and dendritic cells, build up a granuloma, "the hallmark tissue reaction of TB" (Gengenbacher and Kaufmann 2012). Other functions of $\mathrm{CD}^{+}$ Th cells include activation of $\mathrm{CD} 8^{+}$cytotoxic $\mathrm{T}$ cells and B lymphocytes, as well as differentiation into diverse Th cell subtypes.

Depending on the cytokine environment, different types of Th cells will develop. IFN- $\gamma$ drives Th1 cell production and generates clone

\section{BOX 1. DETERMINANTS OF ACQUISITION OF TB INFECTION}

The closeness and duration of contact with a patient with infectious TB (Rieder et al. 2009).

The effective amount of shared air space determined by the level of air circulation and size of the room, which influences effective exposure.

Patients with a higher bacterial load, such as those with cavitatory pulmonary TB and those AFB (acidfast bacillus) smear positive, transmit TB more efficiently.

The susceptibility of the host. 
expansion as well as differentiation into memory cells; on the contrary, IL-10 and IL-4 inhibit their development. Thl cells regulate cell-mediated immunity and activate natural killer cells through IFN- $\gamma$ secretion. High levels of IL-4, IL-5, and IL-10 are needed for the differentiation of Th2 cells, their clonal expansion, and generation of memory cells. IFN- $\gamma$ down-regulates the development of this cell type. Th2 cells are responsible for controlling humoral immunity and activation of B cells into plasma cells.

Adaptive immunity can take as long as $42 \mathrm{~d}$ after MTC exposure and infection to be established (Wallgren 1948; Poulsen 1950; Berry et al. 2010). At this point, a large number of activated macrophages are recruited to the site of infection and construct the granuloma, to which lymphocytes, epithelioid, and giant cells are also attracted. By depriving the center of the granuloma of oxygen, the lowering the $\mathrm{pH}$ necrosis occurs internally, preventing bacterial replication but enabling dormancy. However, it is likely that some replication still occurs, because without this, isoniazid would not be effective against LTBI, as it is only bactericidal against replicating $M$. tuberculosis.

Macrophages and dendritic cells also transport bacteria to the local lymph nodes during the construction of granulomas, allowing the dispersal of infection, particularly to well-oxygenated areas such as lung apices, suprarenal glands, the brain cortex, and bone and growing joints.

\section{Disease}

Only $\sim 10 \%$ of infected individuals develop active disease; of these, most will progress in the first 2 years after exposure. There are two possible methods for the development of active disease-an active primary infection, and reactivation of LTBI. Reactivation may occur years after infection in older individuals, usually caused by a failure of the host immune response (Box 2).

\section{IMMUNOLOGY AND PATHOGENESIS}

The breadth of information available on the immunology and pathogenesis of LTBI means that the intention of this section is not to comprehensively cover all the available material, but rather to provide a summary of the key concepts to allow readers to better understand the diagnostic and therapeutic management of LTBI.

\section{MTC Factors}

M. tuberculosis has a series of tools to enable survival inside the human host. First, a unique cell wall structure with high lipid content protects the bacterium from the highly acidic $\mathrm{pH}$ of the phagosome. Second, it is able to arrest phagosome-lysosome fusion. Third, it interferes in antigen presentation as well as in the normal function of $\mathrm{CD}^{+} \mathrm{T}$ cells, natural killer cells, and the complement attack complex. Fourth, M. tuberculosis can resist host-derived antimicrobials (reactive nitrogen and oxygen intermediates).

\section{Host Factors}

As described above, the human immune response against the MTC includes both the innate defense system (macrophage and natural killer cells, complement activation, ciliary clearance, and secretions of the main airways) and the adaptive response, comprising both cellular and humoral immunity. $\mathrm{T}$ cells are the main component of the cellular response that provides protection against $\mathrm{TB}$ and promotes memory response and granuloma formation. Although historically B cells and humoral immunity were not considered to offer any protection, some studies have shown they do contribute to immunological control (Gupta et al. 2012).

The main benefit of the adaptive response is the presence of immunological memory, so that a copy of each antigen found is stored in memory $\mathrm{T}$ cells and will lead to a rapid and enhanced response to subsequent encounters with that same antigen. This is the basis for the TST, a delayed-type hypersensitivity reaction caused by cytokines released from previously sensitized T cells when they are exposed to PPD antigens. Although hypersensitivity is linked to protective immunity, protection is not complete. Dif- 


\section{BOX 2. RISK FACTORS}

\section{For M. tuberculosis Infection}

- Children are more prone to primary infection, as their immune system is not yet completely mature.

- Close contacts of persons known or suspected of having active TB.

- Health-care workers, as well as residents and employees of congregate settings whose clients are at increased risk for active TB (correctional facilities, homeless shelters).

- Being born in areas that have a high incidence of active TB.

For Progression of Infection to Active Tuberculosis (TB)

- HIV infection

- Treatment with anti-TNF agents

- Solid organ transplantation

- Immunosuppressive treatment

- End-stage renal disease

- Hematological malignancies

- Head and neck neoplasm

- Gastrectomy

- Silicosis

- Underweight and malnutrition

- Corticosteroid therapy

- Diabetes mellitus

- Untreated fibrotic lesions suggesting previous TB in chest X-ray

Some patients have no obvious predisposing factors and may just be naturally susceptible to progression.

ferent studies have shown that TST-positive reactors are less likely to be reinfected than nonresponders, but there is no way to prevent reactivation without specific treatment.

From 2 to 4 wk after the first encounter between bacteria of the MTC and macrophages, the human host prepares two types of responses. The first one is delayed-type hypersensitivity to tubercle bacilli, which is tissue damaging as it promotes destruction of nonactivated macrophages containing proliferating mycobacterium. The second is a cell-mediated response, which activates macrophages to kill bacteria. There is a dynamic equilibrium between these two responses, which can lead to either TB progression or containment of MTC.

\section{BURDEN IMPORTANCE}

In 1993, the World Health Organization (WHO) declared TB to be a global public health emergency, stating that: "Tuberculosis today is humanity's great killer, and it is out of control in many parts of the world. The disease, preventable and treatable, has been grossly neglected and no country is immune to it." The first priority for TB elimination is early diagnosis and treatment of pulmonary TB, to reduce transmission. Subsequent steps include screening for clinically asymptomatic LTBI in high-risk populations. In addition to effective active TB control, the post-2015 WHO global TB strategy recognizes that elimination requires a focus on reducing the 
pool of latently infected individuals from which future TB cases would be generated.

It is estimated that one-third of the world's population is latently infected with MTC. However, given the $10 \%$ progression rate, clinical and public health services must focus their energies primarily on groups at a higher risk of disease. These include recently infected persons, immunocompromised individuals such as HIV (human immunodeficiency virus) patients, antiTNF and transplant candidates, and household contacts of pulmonary TB cases. Additionally, health-care workers and migrants from highincidence countries to low-incidence settings may be tested and given preventive treatment.

\section{DIAGNOSTIC TESTS}

\section{Introduction}

Individuals should not be tested for LTBI unless the responsible clinician has a management plan; "intention to test is intention to treat." The low risk of progression, treatment completion, and effectiveness and the adverse effects of treatment should be taken into account when testing is offered.

As previously stated, there is no way to directly diagnose LTBI, as the MTC cannot be recovered from the host unless active TB is present. An indirect immunological assessment of exposure is made instead by ascertaining the reactivity of host lymphocytes to mycobacterial antigens, either by testing the in vivo response with the TST or in vitro with INF- $\alpha$ release assays (IGRAs).

\section{TST}

The TST consists of an intradermal injection of tuberculin. Although a variety of approaches have been used, the Mantoux technique, which is administered on the inner surface of the forearm, is one of the most widely used globally. Tuberculin material can be PPD or RT-23. The standard doses are 5 PPD units $(0.1 \mathrm{~mL})$ or 2 RT-23 units, which are equivalent. Both types of tuberculins contain a complex mixture of antigens, including those of $M$. bovis-BCG strains and several antigens of nontuberculous mycobacteria (NTM), as well as M. tuberculosis antigens. Tuberculin will stimulate a delayedtype hypersensitivity response via $\mathrm{T}$ lymphocytes. A positive TST can be detected by an induration on the site of injection after $48-72 \mathrm{~h}$. The result is the transverse diameter of the induration, which is usually recorded in millimeters. The interpretation of the result should take into account immunosuppression as well as the prevalence of TB in the setting in question$5 \mathrm{~mm}$ is considered positive if the patient is immunosuppressed, but it will be considered negative in healthy patients living in high-prevalence areas, for whom a $15-\mathrm{mm}$ cutoff will apply. In some settings, a uniform cutoff of $10 \mathrm{~mm}$ is applied. These different and subjective considerations make it difficult to compare TST properties between studies in different countries and populations.

The main limitation of TST is its low specificity because of previous BCG vaccination and NTM infections. There are also other drawbacks in this apparently simple test. In subjects with chronic debilitating conditions or immunosuppression, TST may have a lower sensitivity than in healthy people. It must be administrated and read by trained professionals so as to avoid variability of the results. It also requires two clinical visits, and a positive reaction may interfere with confidentiality issues.

\section{IGRAs}

These tests use a blood sample taken from the patient to measure T-cell release of IFN- $\gamma$ in vitro after stimulation by specific MTC antigens. Two IGRAs have been developed: the QuantiFERON-TB ${ }^{\circledR}$ Gold In-Tube (QFT) (Cellestis Limited, Carnegie, Victoria, Australia) and the T-SPOT.TB ${ }^{\circledR}$ (Oxford Immunotec, Oxford, UK). Both tests use the early secreted antigenic target 6 (ESAT-6) and culture filtrate protein 10 (CFP10), which are encoded by genes located on the region of difference 1 (RD-1) segment of the MTC genome. QFT also includes a third antigen, TB7.7 (RD-11).

QFT is an enzyme-linked immunosorbentassay (ELISA)-based test performed on whole 
blood. It measures levels of IFN- $\gamma$ in the supernatant of a cell suspension. The result is easily processed by software and is reported in international units per $\mathrm{mL}(\mathrm{IU} / \mathrm{mL})$. T-SPOT.TB is an enzyme-linked immunospot assay (ELISPOT) performed on separated $\mathrm{T}$ lymphocytes. The result is reported as number of IFN- $\gamma$ producing $\mathrm{T}$ cells.

The main advantage of IGRAs is their specificity, as ESAT-6, CFP10, and TB7.7 are found in the MTC genome, but not in M. bovis-BCG, or in the majority of NTMs. Moreover, IGRAs can differentiate a negative result from anergy by means of a positive control, which uses phytohemagglutinin to stimulate production of IFN- $\gamma$. In this review, we will talk about IGRAs without distinction, assuming that both QFT and T-SPOT.TB properties are comparable.

Neither TST nor IGRAs are able to detect TB infection in its first stages. Because they measure the adaptive immune response to MTC, an $\sim 8$-wk period after infection is required for a reliable result.

There are three notable disadvantages shared by both types of immunologic tests. The first one is their poor positive predictive value (PPV) for progression to active TB. Several studies have tried to correlate the risk of progression to the quantitative response of both in vivo tests (TST measurement) and in vitro tests (i.e., IFN- $\gamma$ concentration in QFT tubes), but no conclusive results have been found. A recent meta-analysis showed a slightly better PPV with IGRAs compared with TST $(2.4 \%$ for TST and $6.8 \%$ for IGRAs in high-risk groups) for the development of active disease (Diel et al. 2012), i.e., in a group of 100 IGRA-positive subjects tested for LTBI, and left without preemptive treatment, seven individuals would develop TB. However, to date, it is not possible to identify these seven before they progress to active disease. Furthermore, another review concluded that there is little difference between TST and IGRA PPVs (Rangaka et al. 2012), contributing to the WHO deendorsement of the use of IGRAs in developing countries.

The second common disadvantage is the inability of TSTs and IGRAs to distinguish between LTBI and active disease. The WHO has recommended against the use of IGRAs for active TB diagnosis, as a negative result does not rule out disease.

The third issue is that the tests are unable to distinguish recent from remote infection. The response profiles for past and recent infection are not different from each other. Each result must be carefully examined and tailored, as peculiarities of the host may modify sensitivity and specificity of both TST and IGRAs (Abubakar et al. 2013).

\section{Evidence of Diagnostic Accuracy}

\section{Sensitivity}

Because there is no "gold standard" for LTBI diagnosis, it is not possible to properly measure the sensitivity and specificity of the available diagnostic tests. The only individuals with proven $\mathrm{TB}$ infection are active $\mathrm{TB}$ patients, from whom bacteria of the MTC can be isolated. Data from a recent meta-analysis of diagnostic tests in TB patients showed a global sensitivity of 0.70 (TST), 0.81 (QFT), and 0.88 (T-SPOT.TB) (Diel et al. 2010). The immune response of latently infected and active TB patients is likely to not be equivalent, as TB itself is a well-known cause of anergy. Thus, sensitivity may be even higher in healthy people. In this population, a negative TST or IGRA rules out TB infection and has an almost $100 \%$ negative predictive value (NPV) for progression to active TB. A negative result, however, may not be reliable in other clinical scenarios, such as clinical suspicion of active TB or immunosuppressed patients. Several studies have tested how IGRAs perform in these specific circumstances; IGRAs seem to be less affected by immunosuppression than the TST (Redelman-Sidi and Sepkowitz 2013).

\section{Specificity}

Specificity is the main difference between the TST and IGRAs. Tuberculin contains several antigens shared by the MTC, M. bovis-BCG, and NTMs; thus, a positive TST does not necessarily equate to TB infection, especially in BCG-vaccinated subjects, among whom a higher proportion of false-positive results can be found. On 
the contrary, IGRAs have excellent specificity as they use antigens encoded in specific sequences of the MTC genome. Pooled specificity data show a range of $0.86-0.99$ for IGRAs versus 0.97 and 0.59 for TST in BCG-vaccinated and non-BCG-vaccinated individuals, respectively (Diel et al. 2010).

Improved specificity prevents the excessive use of treatment to prevent progression, as falsepositive results will be excluded. If a group of 1000 healthy recent contacts of pulmonary TB cases were tested by TST, $\sim 50 \%$ would test positive and be treated. If an IGRA was used instead, only $30 \%$ would be treated, resulting in large savings by preventing unnecessary treatment.

\section{Other Issues: Two Steps versus One Step and Cost-Effectiveness}

Data on the cost-effectiveness of the different approaches to LTBI diagnosis are limited because of the paucity of information on predictive values. Individually, it is generally more expensive to test with an IGRA than with a TST. In the community, the better the selection of people at risk of TB for testing, the less unnecessary treatment and fewer adverse events, as well as reduced expenditure on testing and follow-up visits.

Guidance on diagnostic strategies for LTBI in individuals at risk varies considerably between countries. Although there are several discrepancies between guidelines in terms of the TST cutoff for positivity, the repetition of TST (looking for a boosting effect), and the use of one or more types of tests, there is growing evidence on the superiority of IGRAs over the TST in terms of sensitivity and specificity. Given their higher specificity and their at least comparable NPV for reactivation of TB, IGRAs may be preferable to TST for targeting people for preventive treatment. There are very few countries (United States, Spain, and Portugal) where the two-step TST is heeded. This strategy aims to obtain a LTBI diagnosis in older and immunosuppressed patients, in which an initial TST may boost the immune system against an older latent infection that would be evident after the second dose of tuberculin. However, the effectiveness of this second test is usually low, and several studies have reported a nonnegligible proportion of false-positive results, mainly in BCG-vaccinated individuals.

A more widely used two-step approach, usually based on cost-effectiveness analysis, is the application of IGRAs to confirm an initial TST. The rationale for this is that the cheaper and potentially equally sensitive test (TST) is applied first, and the diagnosis is confirmed with an IGRA test. Which strategy (IGRAs in place of TST or as a confirmatory test) is more suitable for testing individuals at risk is unclear, but it will depend on the immune status of the host and the expected prevalence of TB infection in the population tested (Table 1). In immunocompetent individuals, in whom the sensitivity of the two tests can be regarded as roughly comparable, positive IGRA results are expected to also be TST-positive. In this scenario, using IGRAs as a confirmatory test may be appropriate if the expected proportion of infection is low. Conversely, if the expected proportion of infection is high, testing with an IGRA instead of TST may be more cost-saving.

In immunocompromised patients, the use of IGRA plus TST to increase sensitivity may avoid missing some infected subjects at a high risk of progression to active disease. However, the results from several studies in patients about to start biologic therapeutics suggest that screening for LTBI and decisions regarding preventive treatment can be reliably based on IGRAs, irrespective of TST results (Laffitte et al. 2009; Chang et al. 2011; Qumseya et al. 2011; Garcovich et al. 2012; Hsia et al. 2012, 2013; Jung et al. 2012; Mariette et al. 2012; Greveson et al. 2013). Furthermore, the additional cost of undertaking two tests for the limited gain in sensitivity likely means that dual testing may not be cost-effective.

\section{TREATMENT}

\section{Drugs - Trial Evidence of Effectiveness}

The treatment of LTBI has a long history, starting from the early studies of the 1950s through to modern trials of novel combination regimens 
Diagnosis and Management of Latent TB Infection

Table 1. Differences between TST and IGRAs according to immune status

\begin{tabular}{|c|c|c|}
\hline & Healthy subjects & $\begin{array}{l}\text { Immunosuppressed subjects (including } \\
\text { HIV, IMID patients attributable to starting } \\
\text { anti-TNF therapy, patients awaiting for } \\
\text { transplantation) }\end{array}$ \\
\hline First step & $\begin{array}{r}\text { Always ru } \\
\text { (Clinical history and examination, ch } \\
\text { culture as well as other tests if extre }\end{array}$ & $\begin{array}{l}\text { le out active TB } \\
\text { est X-ray, and sputum microscopy and } \\
\text { apulmonary TB is suspected) }\end{array}$ \\
\hline When to test? & $\begin{array}{l}\text { Recent infection (contact tracing } \\
\text { study) } \\
\text { Occupational risk (HCW) }\end{array}$ & $\begin{array}{l}\text { At HIV diagnosis } \\
\text { Before the beginning of IS treatment }\end{array}$ \\
\hline How do TST perform? & $\begin{array}{l}\text { Good sensitivity } \\
\text { Poor specificity: high proportion of } \\
\text { false-positive reactions in BCG- } \\
\text { vaccinated subjects } \\
\text { Window period (recent infection) }\end{array}$ & $\begin{array}{l}\text { Less sensitivity because of weakening } \\
\text { condition } \\
\text { Poor specificity: high proportion of false- } \\
\text { positive reactions in BCG-vaccinated } \\
\text { subjects } \\
\text { No window period needed }\end{array}$ \\
\hline How do IGRAs perform? & $\begin{array}{l}\text { Good sensitivity } \\
\text { Good specificity } \\
\text { Window period (recent infection) }\end{array}$ & $\begin{array}{l}\text { Less sensitivity than in healthy subjects, but } \\
\text { better sensitivity than TST } \\
\text { Good specificity } \\
\text { No window period needed }\end{array}$ \\
\hline $\begin{array}{l}\text { Recommended strategies } \\
\text { for LTBI diagnosis and } \\
\text { targeting patients for } \\
\text { preventive treatment }\end{array}$ & $\begin{array}{l}\text { High TB infection prevalence } \\
\text { expected: IGRA } \\
\text { Low TB infection prevalence } \\
\text { expected: two-step strategy } \\
\text { Positive TST followed by an IGRA }\end{array}$ & $\begin{array}{l}\text { Most guidelines recommend TST AND } \\
\text { IGRA to maximize sensitivity; several } \\
\text { studies used ONLY IGRAs with no issues }\end{array}$ \\
\hline
\end{tabular}

TST, tuberculin skin test; IGRA, interferon- $\gamma$ release assay; HIV, human immunodeficiency virus; IMID, immune-mediated inflammatory disease; TNF, tumor necrosis factor; HCW, health-care workers; IS, immunosuppressive; BCG, Bacillus Calmétte-Guérin.

in high-risk populations. Drugs for treating latent infection should possess sterilizing activity against dormant mycobacteria, as such is the status of bacteria in these patients. As previously described, the very fact that isoniazid is active against LTBI suggests that at least some replication is occurring, as the target of this drug's activity is the inhibition of the synthesis of mycolic acid, required for the mycobacterial cell wall, during replication. Numerous randomized controlled trials and a handful of systematic reviews have shown the safety and efficacy of isoniazid in the general population, HIV-infected individuals, and posttransplant patients for preventing $\mathrm{TB}$ reactivation.

Rifampicin, the second proven active drug for LTBI, is a well-known antibiotic with intracellular activity that inhibits the bacterial DNAdependent RNA polymerase and has been used against other bacterial infections in their latency period.

Globally, it is most common to use 6-9 mo of daily isoniazid monotherapy (dose $300 \mathrm{mg}$ ) although recent data suggest that 4 mo of daily rifampicin (dose $600 \mathrm{mg}$ ), or 3 mo of combined therapy with isoniazid $300 \mathrm{mg}$ and rifampicin $600 \mathrm{mg}$ may be equally effective (Stagg et al. 2014). A novel regimen consisting of 12 doses of a weekly regimen of rifapentin $(900 \mathrm{mg}$ ) and high-dose isoniazid $(900 \mathrm{mg}$ ) has been recently described as effective and is currently used in the United States under directly observed therapy.

The first and major reason for treatment failure, and thus for the development of active $\mathrm{TB}$, is poor medication adherence. Unfortunately, the direct observation of each patient is unaffordable for most countries. LTBI treat- 
ments are lengthy, and a close monitoring of subjects is required to ensure high treatment compliance. Several methods for checking treatment compliance have been validated. They include the Eidus-Hamilton test and commercially available reactive strips that can easily detect urine metabolites of isoniazid metabolites. Checking the orange-colored urine of subjects taking rifampicin may also be useful for adherence control.

\section{Adverse Events}

The three common treatment regimens are generally well tolerated. Patients must always be told about symptoms that may indicate the development of adverse effects so that they seek care promptly. The most common life-threatening adverse event resulting from isoniazid is liver toxicity. Not only is a clinical assessment needed, but liver function tests are also essential in the third or fourth week of treatment, and then in the second and third month, as well as in the instance of patients reporting hepatitis signs and symptoms. Biochemistry variations on alanine transaminase (ALT) and aspartate transaminase (AST) precede symptoms as indicators of liver damage and that is the reason for testing apparently healthy patients. Another frequent adverse event of isoniazid is peripheral neuropathy, which can easily be prevented with daily pyridoxine.

Patients taking rifamycins can present flulike symptoms in their first week of treatment. This adverse event is more common in intermittent regimens (i.e., weekly doses).

Hypersensitivity reactions are more frequently related to rifamycins, although they might also be caused by isoniazid. These reactions are mostly seen in the second to fourth week of treatment and consist of a rash and fever, ranging from harmless itching of the skin to severe clinical pictures including Stevens-Johnson syndrome.

\section{Special Issues}

Although preventing drug-resistant TB cases is of great concern for clinicians and health au- thorities, there are no recommended regimens for LTBI treatment of those exposed to multidrug-resistant $\mathrm{TB}$, as there are no sterilizing drugs for dormant bacilli apart from isoniazid and the rifamycins. Guidelines advise only "watchful waiting" of these individuals to assure an early diagnosis in case of the development of active TB. Young children, who are household contacts of multidrug-resistant TB cases, may benefit from prophylactic treatment. This should always be supervised by a physician with knowledge and experience of multidrug-resistant TB prophylactic treatment, based on drug susceptibility results of the index case.

For all LTBI patients diagnosed within a contact study, it is mandatory for the clinician to check the drug susceptibility of the index case and to ensure that the transmitted bacteria are likely to be sensitive to the chosen LTBI treatment regimen.

\section{MANAGEMENT}

The most important issue for LTBI management is to properly target candidates for screening and treating, that is, to identify an individual's risk of developing active TB. The main contraindication for LTBI treatment is active $\mathrm{TB}$, and thus the first commitment of the clinician is to rule out the disease through a clinical interview for the specific symptoms of TB and a careful examination of a recent chest X-ray. Medical and nursing staff skilled in treating TB patients and dealing with adverse events of antituberculosis drugs should ideally manage LTBI screening.

\section{Individuals at Risk}

\section{Newly Infected Individuals}

As mentioned above, the first 2 yr after infection carry the highest risk for developing active disease. For this reason, individuals at risk for MTC infection found to have converted from a negative to a positive result for a LTBI diagnostic test, as well as individuals testing positive after a contact tracing study, will be the most likely to benefit from preventive treatment. 
Contact Tracing (Erkens et al. 2010). After diagnosing an individual with pulmonary TB, it is mandatory to get information regarding transmission settings to assign priorities for the investigation. The purpose of conducting a contact examination of a TB case is to evaluate individuals for active TB disease (to find either the source case or secondary cases) so that treatment can be given and further transmission stopped, as well as diagnosing and treating those with LTBI.

Priorities for contact investigation depend on the characteristics of the index case (cavitation on chest X-ray and positive AFB smear sputum are the most infectious), susceptibility and vulnerability of contacts (children under $5 \mathrm{yr}$ and immunocompromised patients are assigned the highest priority), and circumstances of the exposure (household contacts, congregate settings).

All referred contacts should be specifically asked for respiratory symptoms and current general health. Then, a TST or an IGRA (either alone or in a two-step strategy) should be undertaken. Patients with negative results obtained before $8 \mathrm{wk}$ after the end of exposure should have the test repeated so as to avoid false-negative results in the window period.

Subjects reporting any suggestive symptom of TB and all individuals with positive results on the LTBI diagnostic tests should have a chest Xray to rule out active TB. After TB is excluded, all LTBI patients should be offered treatment with either isoniazid (6 mo), rifampicin (4 mo), or a combined 3-mo regimen with daily isoniazid and rifampicin or weekly isoniazid and rifapentine, with close follow-up. This includes adherence, asking for symptoms attributable to adverse events, and liver function tests, with at least one test before the beginning of treatment and another in 3-4 wk. Further tests may be needed.

Household contacts who are children (or those with a close relationship with the TB patient) should have $\mathrm{TB}$ ruled out with a chest X-ray. They should also be started on preventive treatment even though a negative result may be found in the first LTBI test (TST or an IGRA). Children, especially those under 5 yr of age, have an immature immune system that fa- vors both false-negative results and the development of active TB. If active TB has been excluded and a second LTBI diagnostic test remains negative after the window period, treatment can be stopped.

When a child is found to have TB disease, a contact study should be performed around them, because although TB in children is rarely infectious, it is usually a sign of a close contact who is the source case.

Health-Care Workers. An IGRA or TST may be used for persons with occupational exposure to the MTC. If TB infection is systematically tested for and ruled out when the individual commences their new job, and then every $2 \mathrm{yr}$ as long as they remain negative responders, a conversion from negative to positive result may be detected and thus treatment for a recent infection could be offered to prevent TB development.

\section{Immunocompromised Patients}

Immunocompromised persons with LTBI are at an increased risk for progression to active TB; treatment of LTBI in this population can reduce this risk.

People Living with HIV. Patients coinfected with HIV and MTC are particularly prone to a reactivation of LTBI and the development of disseminated disease. For this reason, systematic screening for $\mathrm{TB}$ infection in individuals newly diagnosed with HIV has been recommended. After ruling out active disease, patients should be tested with either a TST or an IGRA, and those with positive results should receive preventive treatment. Several studies have shown the better performance of IGRAs over TSTs in patients with lower CD4 cells counts $\left(<200\right.$ cells $\left./ \mathrm{mm}^{3}\right)$. These patients should be treated with at least $6 \mathrm{mo}$ of isoniazid $300 \mathrm{mg}$ daily.

Subjects Considered for Anti-TNF Drugs. Individuals diagnosed with immune-mediated inflammatory diseases have an impaired immune system attributable to their disease or its treatment. Those receiving anti-TNF (especially infliximab and adalimumab) are at particular risk for reactivation, as these drugs break up 
granulomas, which are partially maintained by self-released TNF.

One strategy proposed in candidates to receive anti-TNF is systematically screening for LTBI before treatment begins. How to test this population is still an area of debate in different countries. To ensure the greatest sensitivity, several nations have encouraged the use of both TST and an IGRA and the treatment of any patient with a positive result.

Transplant Candidates. Among solid organ transplant recipients, the risk of developing active TB has been calculated to be 36- to 74-fold higher than in the general population (Singh and Paterson 1998), with lung transplantation being the most risky. Active screening of LTBI is increasingly becoming part of the routine in the check-up of transplant candidates before the surgical procedure.

Patients with end-stage kidney disease and those with advanced liver cirrhosis waiting for kidney and liver transplantation deserve special comment. These two conditions are risk factors for skin test anergy and thus an increased rate of false-negative results in TST. For this reason, an IGRA is preferred to determine which patients should receive LTBI treatment.

\section{Migrants from Endemic Areas Arriving to High-Income Countries}

As the majority of TB in migrants arises through the reactivation of infections acquired overseas and developed in the first few years postentry, screening for LTBI in this population on arrival is an essential element of any TB elimination strategy. This group may be a hard-to-reach population, with low rates of returning to have TSTs read; thus, an IGRA test might be preferred. The use of IGRAs can increase test completion rates, so control efforts should focus on those most likely to benefit from further evaluation and treatment.

\section{LOOKING TO THE FUTURE}

The control and eventual global elimination of TB would depend on either an effective postexposure vaccine or better tools and drugs to detect and treat LTBI. Current research focused on improving the diagnosis and positive predictive value of LTBI tests ranges from improvements of IGRAs by investigating additional antigens as well as alternative cytokines, to research based on proteomics, transcriptomics, and metabolomics. For example, several ongoing studies in humans are measuring different cytokine profiles (e.g., IFN- $\gamma$ and IL2) to differentiate active TB and LTBI in vitro, whereas others have focused on transcriptional profiles apparently associated with latently infected individuals who might progress to disease. It is likely that advances in our understanding of latency in TB would be revolutionized in the next decade based on ongoing research in large-population cohorts using these approaches.

New research must also identify treatment regimens that are considerably shorter, more effective, and less toxic. The new rifapentine regimen of only 12 doses has been made possible because of the long half-life of this drug. Future research must consider whether newer drugs with a long half-life, such as bedaquiline, may be used to shorten treatment. Such research must also investigate the adverse effect profile of any new regimen.

\section{REFERENCES}

Abubakar I, Stagg HR, Whitworth H, Lalvani A. 2013. How should I interpret an interferon $\gamma$ release assay result for tuberculosis infection? Thorax 68: 298-301.

Berry MP, Graham CM, McNab FW, Xu Z, Bloch SA, Oni T, Wilkinson KA, Banchereau R, Skinner J, Wilkinson RJ, et al. 2010. An interferon-inducible neutrophil-driven blood transcriptional signature in human tuberculosis. Nature 466: 973-977.

Calmette A. 1931. Preventive vaccination against tuberculosis with BCG. Proc R Soc Med 24: 1481-1490.

Chang B, Park HY, Jeon K, Ahn JK, Cha HS, Koh EM, Kang ES, Koh WJ. 2011. Interferon- $\gamma$ release assay in the diagnosis of latent tuberculosis infection in arthritis patients treated with tumor necrosis factor antagonists in Korea. Clin Rheumatol 30: 1535-1541.

Diel R, Loddenkemper R, Nienhaus A. 2010. Evidencebased comparison of commercial interferon- $\gamma$ release assays for detecting active TB. A meta analysis. Chest 137: 952-968.

Diel R, Loddenkemper R, Nienhaus A. 2012. Predictive value of interferon- $\gamma$ release assays and tuberculin skin testing for progression from latent TB infection to disease state: A meta-analysis. Chest 142: 63-75. 
Diagnosis and Management of Latent TB Infection

Erkens CGM, Kamphorst M, Abubakar I, Bothamley GH, Chemtob D, Haase W, Migliori GB, Rieder HL, Zellweger JP, Lange C. 2010. Tuberculosis contact investigation in low prevalence countries: A European consensus. Eur Respir J 36: 925-949.

Garcovich S, Ruggeri A, D’Agostino M, Ardito F, De Simone C, Delogu G, Fadda G. 2012. Clinical applicability of Quantiferon-TB-Gold testing in psoriasis patients during long-term anti-TNF- $\alpha$ treatment: A prospective, observational study. J Eur Acad Dermatol Venereol 26: 15721576.

Gengenbacher M, Kaufmann SH. 2012. Mycobacterium tuberculosis: Success through dormancy. FEMS Microbiol Rev 36: 514-532.

Greveson K, Goodhand J, Capocci S, Woodward S, Murray C, Cropley I, Hamilton M, Lipman M. 2013. Yield and cost effectiveness of mycobacterial infection detection using a simple IGRA-based protocol in UK subjects with inflammatory bowel disease suitable for antiTNF $\alpha$ therapy. J Crohn's Colitis 7: 412-418.

Gupta A, Kaul A, Tsolaki AG, Kishore U, Bhakta S. 2012. Mycobacterium tuberculosis: Immune evasion, latency and reactivation. Immunobiology 217: 363-374.

Hillier A. 1903. The nature of the infectivity of phthisis: A study of the views of Koch, Flügge, and others. Br Med J 1: 593-596.

Hsia EC, Schluger N, Cush JJ, Chaisson RE, Matteson EL, Xu S, Beutler A, Doyle MK, Hsu B, Rahman MU. 2012. Interferon- $\gamma$ release assay versus tuberculin skin test prior to treatment with golimumab, a human anti-tumor necrosis factor antibody, in patients with rheumatoid arthritis, psoriatic arthritis, or ankylosing spondylitis. Arthritis Rheum 64: 2068-2077.

Hsia EC, Cush JJ, Matteson EL, Beutler A, Doyle MK, Hsu B, Xu S, Rahman MU. 2013. Comprehensive tuberculosis screening program in patients with inflammatory arthritides treated with golimumab, a human anti-tumor necrosis factor antibody, in Phase III clinical trials. Arthritis Care Res (Hoboken) 65: 309-313.

Jung YJ, Lyu J, Yoo B, Lee CK, Kim YG, Yang SK, Byeon JS, Kim KJ, Ye BD, Lee KH, et al. 2012. Combined use of a TST and the T-SPOT ${ }^{\circledR}$.TB assay for latent tuberculosis infection diagnosis before anti-TNF- $\alpha$ treatment. Int $J$ Tuberc Lung Dis 16: 1300-1306.

Koch R. 1891. A further communication on a remedy for tuberculosis. Br Med J 1: 125-127.

Laffitte E, Janssens JP, Roux-Lombard P, Thielen AM, Barde C, Marazza G, Panizzon RG, Saurat JH. 2009. Tubercu- losis screening in patients with psoriasis before antitumour necrosis factor therapy: Comparison of an interferon- $\gamma$ release assay vs. tuberculin skin test. $\mathrm{Br} J$ Dermatol 161: 797-800.

Mariette X, Baron G, Tubach F, Lioté F, Combe B, MiceliRichard C, Flipo RM, Goupille P, Allez M, Salmon D, et al. 2012. Influence of replacing tuberculin skin test with ex vivo interferon $\gamma$ release assays on decision to administer prophylactic antituberculosis antibiotics before anti-TNF therapy. Ann Rheum Dis 71: 1783-1790.

Martin CF, Robins GD. 1898. On the diagnostic value of tuberculin. Br Med J 1: 357-359.

Mi'fadyean J. 1891. Experiments with tuberculin on cattle. Br Med J 1: 634-635.

Poulsen A. 1950. Some clinical features of tuberculosis. Acta Tuberc Scand 24: 311-346.

Qumseya BJ, Ananthakrishnan AN, Skaros S, Bonner M, Issa M, Zadvornova Y, Naik A, Perera L, Binion DG. 2011. QuantiFERON-TB Gold testing for tuberculosis screening in an inflammatory bowel disease cohort in the United States. Inflamm Bowel Dis 17: 77-83.

Rangaka MX, Wilkinson KA, Glynn JR, Ling D, Menzies D, Mwansa-Kambafwile J, Fielding K, Wilkinson RJ, Pai M. 2012. Predictive value of interferon- $\gamma$ release assays for incident active tuberculosis: A systematic review and meta-analysis. Lancet Infect Dis 12: 45-55.

Redelman-Sidi G, Sepkowitz KA. 2013. IFN- $\gamma$ release assays in the diagnosis of latent tuberculosis infection among immunocompromised adults. Am J Respir Crit Care Med 188: $422-431$.

Rieder H, Chen-Yuan C, Gie RP, Enarson D. 2009. General background to clinical tuberculosis. Infection and disease. Crofton's clinical tuberculosis, 3rd ed., pp. 1-10. Macmillan, Oxford.

Singh N, Paterson D. 1998. Mycobacterium tuberculosis infection in solid-organ transplant recipients: Impact and implications for management. Clin Infect Dis 27: 12661277.

Stagg HR, Zenner D, Harris RJ, Muñoz L, Lipman MC, Abubakar I. 2014. Treatment of latent tuberculosis: A network meta-analysis. Ann Intern Med (in press).

Wallgren A. 1948. The time-table of tuberculosis. Tubercle 29: $245-251$.

Wang L, Turner MO, Elwood RK, Schulzer M, FitzGerald JM. 2002. A meta-analysis of the effect of Bacille Calmette Guérin vaccination on tuberculin skin test measurements. Thorax 57: 804-809. 